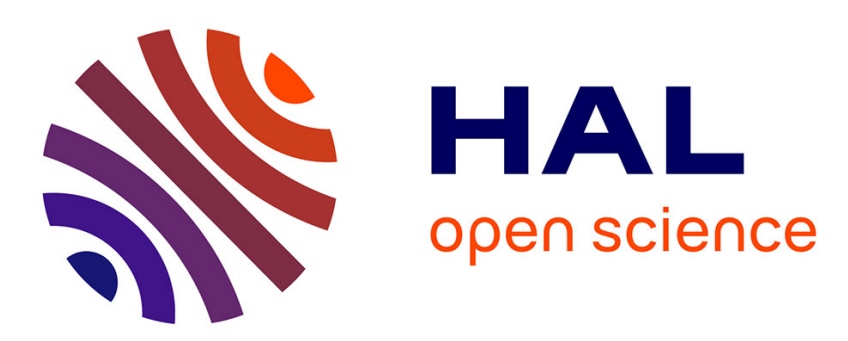

\title{
Making the Planetary Material Diversity during the Early Assembling of the Solar System
}

Francesco C Pignatale, Sébastien Charnoz, Marc Chaussidon, Emmanuel

Jacquet

\section{- To cite this version:}

Francesco C Pignatale, Sébastien Charnoz, Marc Chaussidon, Emmanuel Jacquet. Making the Planetary Material Diversity during the Early Assembling of the Solar System. The Astrophysical journal letters, 2018, 867 (2), pp.L23. 10.3847/2041-8213/aaeb22 . insu-02900905

\section{HAL Id: insu-02900905 \\ https://hal-insu.archives-ouvertes.fr/insu-02900905}

Submitted on 6 Aug 2020

HAL is a multi-disciplinary open access archive for the deposit and dissemination of scientific research documents, whether they are published or not. The documents may come from teaching and research institutions in France or abroad, or from public or private research centers.
L'archive ouverte pluridisciplinaire HAL, est destinée au dépôt et à la diffusion de documents scientifiques de niveau recherche, publiés ou non, émanant des établissements d'enseignement et de recherche français ou étrangers, des laboratoires publics ou privés. 


\title{
Making the Planetary Material Diversity during the Early Assembling of the Solar System
}

\author{
Francesco C. Pignatale ${ }^{1,2}$ (10), Sébastien Charnoz ${ }^{1}$ (10), Marc Chaussidon ${ }^{1}$, and Emmanuel Jacquet ${ }^{2}$ \\ ${ }^{1}$ Institut de Physique du Globe de Paris (IPGP) 1 rue Jussieu, F-75005, Paris, France; pignatale @ipgp.fr \\ ${ }^{2}$ Muséum national d'Histoire naturelle, UMR 7590, CP52 57 rue Cuvier, F-75005, Paris, France \\ Received 2018 August 13; revised 2018 October 15; accepted 2018 October 24; published 2018 November 5
}

\begin{abstract}
Chondritic meteorites, the building blocks of terrestrial planets, are made of an out-of-equilibrium assemblage of solids formed at high and low temperatures, either in our Solar system or previous generations of stars. For decades this was considered to result from large-scale transport processes in the Sun's isolated accretion disk. However, mounting evidence suggests that refractory inclusions in chondrites formed contemporaneously with the disk building. Here we numerically investigate, using a 1D model and several physical and chemical processes, the formation and transport of rocky materials during the collapse of the Sun's parent cloud and the consequent assembling of the Solar Nebula. The interplay between the cloud collapse, the dynamics of gas and dust, vaporization, recondensation, and thermal processing of different species in the disk results in a local mixing of solids with different thermal histories. Moreover, our results also explain the overabundance of refractory materials far from the Sun and their short-formation timescales, during the first tens of kyr of the Sun, corresponding to class 0 -I, opening new windows into the origin of the compositional diversity of chondrites.
\end{abstract}

Key words: meteorites, meteors, meteoroids - protoplanetary disks - stars: formation

\section{Introduction}

Chondritic meteorites are fragments of planetesimals formed in the Solar Nebula within the first $4 \mathrm{Myr}$ of Solar System history (the time 0 being the formation of the highly refractory Ca- and Al- rich inclusions (CAIs); Chaussidon \& Liu 2015). They are made of a mixture of materials that formed at high and low temperatures (Scott \& Krot 2003). This peculiar assemblage has always been puzzling and is one key ingredient in the composition of terrestrial planets. A striking paradox is that carbonaceous chondrites, which presumably accreted in the outer solar system at temperatures lower than $150 \mathrm{~K}$ (Warren 2011), are the richest in CAIs formed at temperatures higher than $1600 \mathrm{~K}$, presumably close to the Sun (Scott \& Krot 2003). The chemical composition of CAIs links them to precursors condensed from the Solar gas (Richter et al. 2006).

Absolute $\mathrm{Pb}-\mathrm{Pb}$ ages show that CAIs in carbonaceous chondrites were formed over a brief interval (time, $t<160 \mathrm{kyr}$ ) at the very beginning of the Solar System (Connelly et al. 2012) from refractory precursors that, according to short-lived ${ }^{26} \mathrm{Al}$ chronologies, could have condensed over an even shorter period of 10-20 kyr (Jacobsen et al. 2008; Larsen et al. 2011; Mishra \& Chaussidon 2014). Such a timescale is shorter than the assembling time of a protoplanetary disk from the infalling molecular cloud (Williams \& Cieza 2011). Thus it seems unavoidable that the formation of the earliest solar system solids (CAIs at least) occurred concurrently with the building of the Solar Nebula, in agreement with observations of young stellar objects showing that dust grows and becomes largely crystalline as soon as the star begins to form (Williams \& Cieza 2011; Chiang et al. 2012). Because increasing observational and theoretical evidence shows that disks start off compact (Dullemond et al. 2006; Jacquet et al. 2011; Hennebelle et al. 2016; Cox et al. 2017), most of the infalling material should be located initially close to the protostar and undergoes thermal processing before incorporation into planetary material, like chondrites or planetesimals.
To understand the origin of this material, we have developed a 1D simulation to track the formation of the disk and the dust thermal evolution during the collapse of the parent cloud.

\section{Methods and Models}

The code aims at tracking the $1 \mathrm{D}$ evolution of a protoplanetary disk with different gaseous and solid species. We use a simplified treatment of the gas and dust dynamics and chemistry (nevertheless including a variety of relevant coupled processes) in order to unveil key physical processes associated with the coupling of transport and thermal processing in a connected cloud-disk system. This is why the infalling envelope is described as a spherical isothermal shell collapsing while conserving angular momentum, in order to make the calculations computationally tractable (see e.g., Hueso \& Guillot 2005). Although more detailed models are available for the cloud collapse, for example Hennebelle et al. (2016), and there are numerous the studies investigating the disk chemistry (Henning \& Semenov 2013), our approach represents the first attempt to implement in one single simulation several dynamical and chemical aspects of cloud collapse and disk formation and evolution.

The code is a classic alpha-disk model built on the model of Hueso \& Guillot (2005) that considers cloud collapse and radiative and viscous heating. Our 1D disk is described by a logarithmic grid (100 cells, between 0.01 and $1000 \mathrm{au}$ ). We include gravitational instability, dead zone (DZ), in the form of a parameterized description (Terquem 2008). We use a layered accretion model of Zhu et al. (2010) with a low- $\alpha$ midplane and high- $\alpha$ surface layers, dust transport through advection and diffusion, and growth/fragmentation using the method described by Drążkowska et al. (2016) and Birnstiel et al. (2012). The opacity is calculated using the opacity table from Baillié et al. (2016) and the procedures described in Helling et al. (2000) and Semenov et al. (2003). Starting from the work of Yang \& Ciesla (2012) on refractory dust, we now include 
Table 1

Simple Chemistry Rules Implemented in the Code

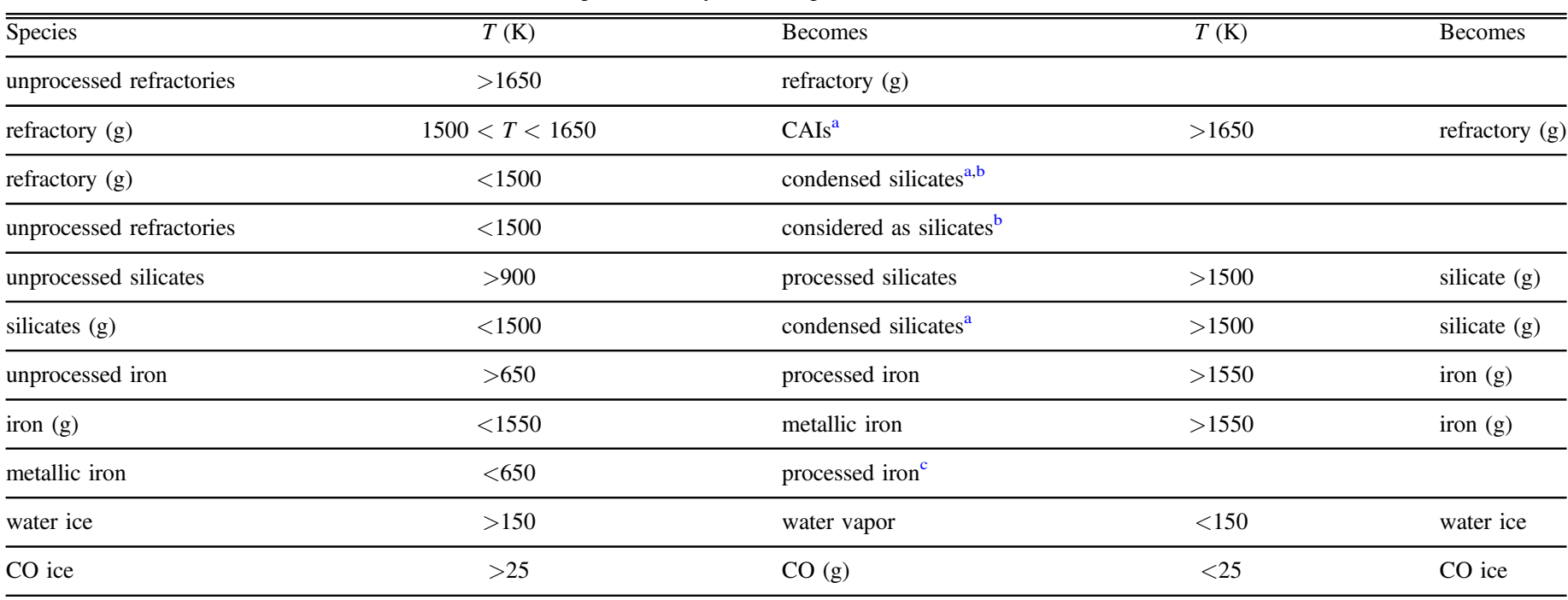

$\mathrm{H}_{2}(\mathrm{~g})$

Notes. We assume that the kinetics timescales of evaporation, condensation, and processing are instantaneous or have kinetics that are close to the timesteps used in the calculations, $\delta t 10^{-2}$ yr.

${ }^{a}$ In our calculations condensed CAIs and condensed silicates do not equilibrate with the surrounding environment as evidenced by actual chondrites.

$\mathrm{b}$ This allows us to distinguish between the most refractory Ca-Al-bearing phases (such as hibonite and melilite) that condense at $1500<T(\mathrm{~K})<1650$ (those we are more interested in) and the other less refractory Ca- and Al-bearing silicates (like the fassaite phase) that condense at $T<1500 \mathrm{~K}$ together with the main silicates (Pignatale et al. 2011). As a consequence, our estimation of CAIs production can be considered conservative.

${ }^{c}$ Metallic iron is defined processed at $T<650 \mathrm{~K}$ as it can undergo chemical alterations such as sulfidation and oxidation (Barshay \& Lewis 1976; Ebel 2006).

multiple chemical species allowing material condensation and sublimation using a simple chemical model.

The infall of the parent cloud is described as a source term and we describe the main aspects here. The cloud collapses onto the protostar-disk system with a constant accretion rate (Shu 1977)

$$
\dot{M}=0.975 \frac{C_{\mathrm{cd}}^{3}}{G},
$$

where $G$ is the gravitational constant, and $C_{\mathrm{cd}}$ is the isothermal sound speed $^{3}$ in the cloud. Assuming that the angular momentum of the cloud material is conserved during the infall, the surface density accreted below a threshold radius, called "centrifugal radius," is (Hueso \& Guillot 2005; Yang \& Ciesla 2012)

$$
\dot{\sigma}(r, t)=\frac{\dot{M}}{8 \pi R_{c}^{2}}\left(\frac{r}{R_{c}(t)}\right)^{-3 / 2}\left[1-\left(\frac{r}{R_{c}(t)}\right)^{1 / 2}\right]^{-1 / 2},
$$

where the centrifugal radius $R_{c}(t)$ is the radius under which the cloud collapses onto the disk. $R_{c}(t)$ is initially so small that all infalling material falls directly onto the protostar. As the star mass increases, $R_{c}(t)$ increases and the cloud material progressively feeds the disk. $R_{c}(t)$ is determined to be the place where the specific angular momentum of the infalling material is equal to the specific angular momentum of the Keplerian disk. Thus, deeper cloud envelopes fall close to the protostar, while exterior cloud envelopes fall far from the

\footnotetext{
$3 \quad C_{\mathrm{cd}}^{2}=k_{b} T_{\mathrm{cd}} / \mu m_{p}$ where $k_{b}$ is the Boltzmann constant, $T_{\mathrm{cd}}$ is the cloud temperature, and $\mu=2.2$ is the molecular weight of the gas in terms of the proton mass, $m_{p}$.
}

protostar. The expression for the centrifugal radius is

$$
R_{c}(t)=53\left(\frac{\omega_{\mathrm{cd}}}{10^{-14} \mathrm{~s}^{-1}}\right)^{2}\left(\frac{T_{\mathrm{cd}}}{10 \mathrm{~K}}\right)^{4}\left(\frac{M(t)}{1 M_{\odot}}\right)^{3},
$$

where $\omega_{\text {cd }}$ is the constant angular velocity of the cloud, $T_{\text {cd }}$ is the temperature of the cloud, and $M(t)$ is the total mass of the protostar+disk system at time $t$.

Concerning the chemistry, a simplified approach is used where simple phase transitions are considered. Using a thermochemical code to compute in real time the exact chemical evolution of the different species in the simulation is very challenging. Indeed, at each time step and location the local gas and dust composition changes. This implies the needs for implementing and tracking at least several dozens of compounds if only a short list of elements were considered (see, for example, the shortened list of 60 species that can be stable in protoplanetary disk conditions derived in Pignatale et al. 2011 using the 15 most abundant of the Sun's elements; Asplund et al. 2009).

Consequently, we introduce a simplified chemical model (but nevertheless physically motivated, as the temperatures of condensation/processing of numerous species are well established; Barshay \& Lewis 1976; Ebel 2006) in order to preserve the main trends of the chemistry. In our simulations, the bulk chemical composition of the parent cloud is assumed to be solar (Asplund et al. 2009; Pignatale et al. 2011), with all of the major rock-forming elements $(\mathrm{Si}, \mathrm{Mg}, \mathrm{Al}, \mathrm{Ca}, \mathrm{Fe}$ ) hosted in interstellar unprocessed solid phases. We allow the infalling interstellar materials to be either vaporized, processed (i.e., heated at temperatures $T>900 \mathrm{~K}$ enough to thermally alter the dust and destroy presolar grains but below vaporization), or unaltered upon its arrival in the disk. 


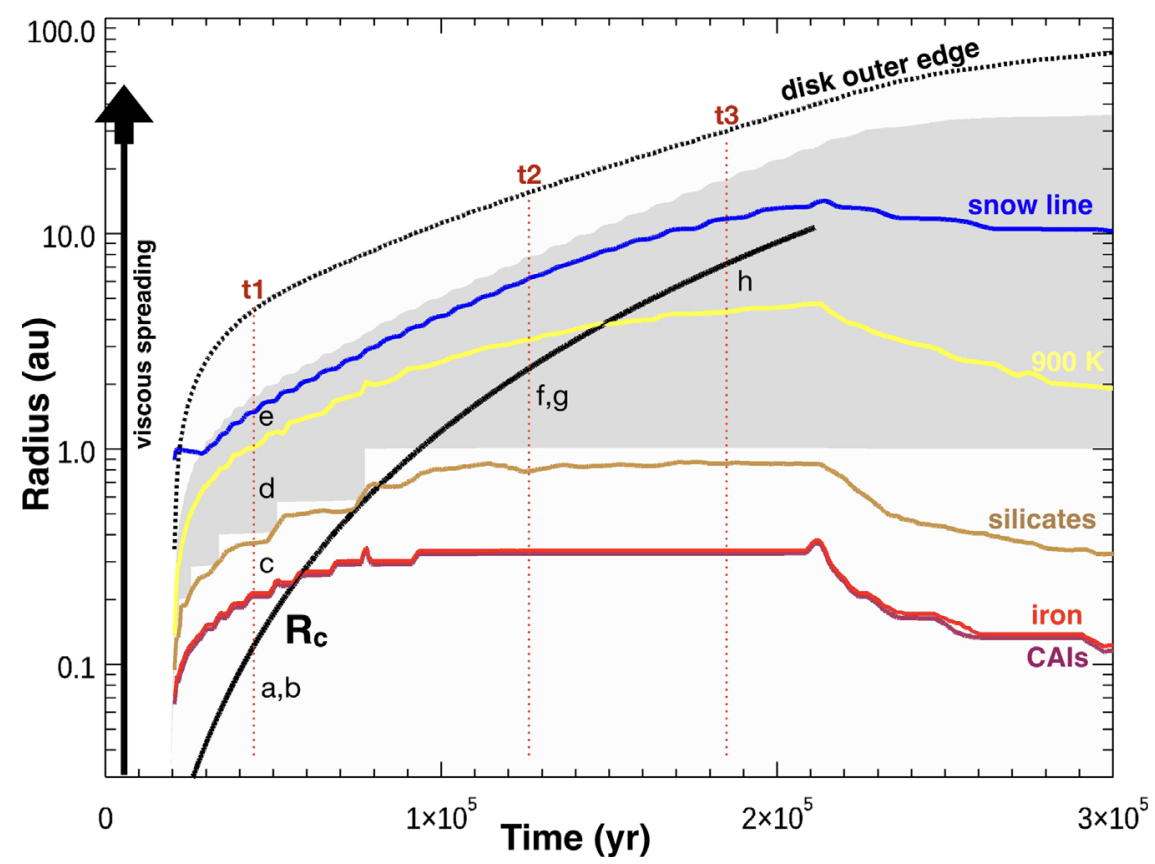

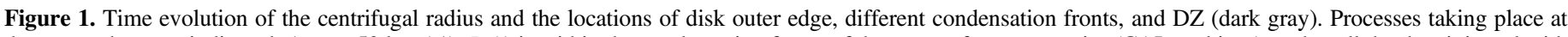

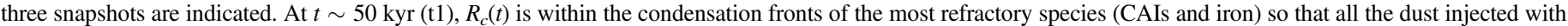

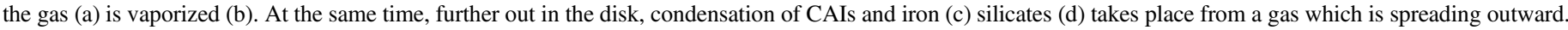

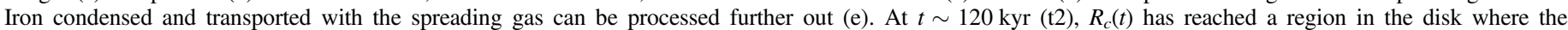

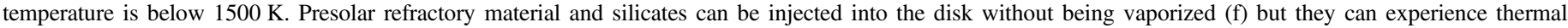

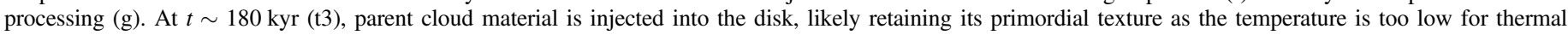
processing (h).

The model tracks the formation and transport in the disk of gaseous species and different solid species with their own vaporization temperatures (see Table 1: (i) condensed refractory phases, which are the potential precursors of CAIs present in meteorites, hereafter called CAIs for the sake of simplicity (ii) condensed metal named hereafter metallic iron, (iii) condensed silicates, (iv) thermally processed and unprocessed silicate dust, (v) thermally processed and unprocessed iron dust, (vi) water ice, and (vii) $\mathrm{CO}$ ice. Molecular hydrogen is then the main gas species.

Chondrule formation is neglected, as we essentially model their precursors. However, chondrule formation is not expected to affect large-scale compositional fractionations in the disk (Goldberg et al. 2015). Heating from accretion shock is also neglected because of the low temperatures involved (Dominik \& Dullemond 2008).

\section{Results and Discussion}

Figure 1 shows the time evolution of the centrifugal radius and the locations of disk outer edge, different condensation fronts, and DZ (dark gray). With our initial conditions $\left(T_{\mathrm{cd}}=15 \mathrm{~K}, \quad \Omega_{\mathrm{cd}}=10^{-14} \mathrm{rad} \mathrm{s}^{-1}, \quad M_{0, \star}=0.02 M_{\odot}, \quad T_{\star}=\right.$ $4000 \mathrm{~K}, \quad R_{\star}=3 R_{\odot}, L_{\star}=$ constant, $M_{\text {tot }}=1 M_{\odot}, \quad \alpha_{\text {active }}=$ $10^{-2}, \alpha_{\text {dead }}=10^{-5}, v_{\text {frag }}=10 \mathrm{~ms}^{-1}$ ), the cloud infall and disks assemblage lasts $\sim 220 \mathrm{kyr}$. While being fed, the disks also expand due to viscous spreading (Hueso \& Guillot 2005; Yang \& Ciesla 2012). After $t \sim 220 \mathrm{kyr}$ the material starts flowing mostly inward as the disk accretes onto the proto-Sun. During the infall, a DZ, a region of low diffusivity (Terquem 2008; Turner \& Sano 2008), develops between $\sim 0.1$ and $\sim 10$ au.
As the dust is incorporated into the disk, its composition changes because of heating of minerals with different sublimation temperatures. Our simulations show that the final distribution of minerals is controlled by the interplay between two key parameters: (i) the centrifugal radius, $R_{c}(t)$, i.e., the distance (increasing with time) within which the parent cloud material is injected, and (ii) the location of the different condensation fronts and properties of the different mineral species, parameters never fully considered previously. Because of angular momentum constraints, $R_{c}(t)$ is initially close to the forming Sun fed from the parent cloud's innermost regions (Kimura et al. 2016). Then $R_{c}(t)$ increases with time due to the arrival of originally more distant cloud materials with a higher specific angular momentum. Under our initial conditions, $R_{c}(t)$ increases from 0 to $\sim 10$ au when the infall ends (Figure 1). The location of the condensation fronts of the different solid species considered depends on the disk temperature profile which is, itself, controlled by viscous and radiative heating (Charnoz et al. 2018). For a given species, if $R_{c}(t)$ is inside of the condensation front of that species, then the dust will be totally vaporized upon its injection into the disk. However, the disk spreads outward concurrently, so that gas injected at high temperature cools down while moving outward, allowing its condensation at larger heliocentric distance. Moreover, dust injected at a later stage, in a lower temperature environment, can be just processed without being vaporized, or survive thermally unaltered. These processes are illustrated in Figure 1.

Figures 2 and 3 show four time-snapshots of the surface density profiles of gas dust and ices and the radial distribution of the mass fraction of the considered rocky material. Our results show that at $t \sim 40 \mathrm{kyr}$ (Figure 3(a)) the disk is only populated by condensed dust (CAIs, iron, and silicates) and only one kind of processed dust: condensed metallic iron that 

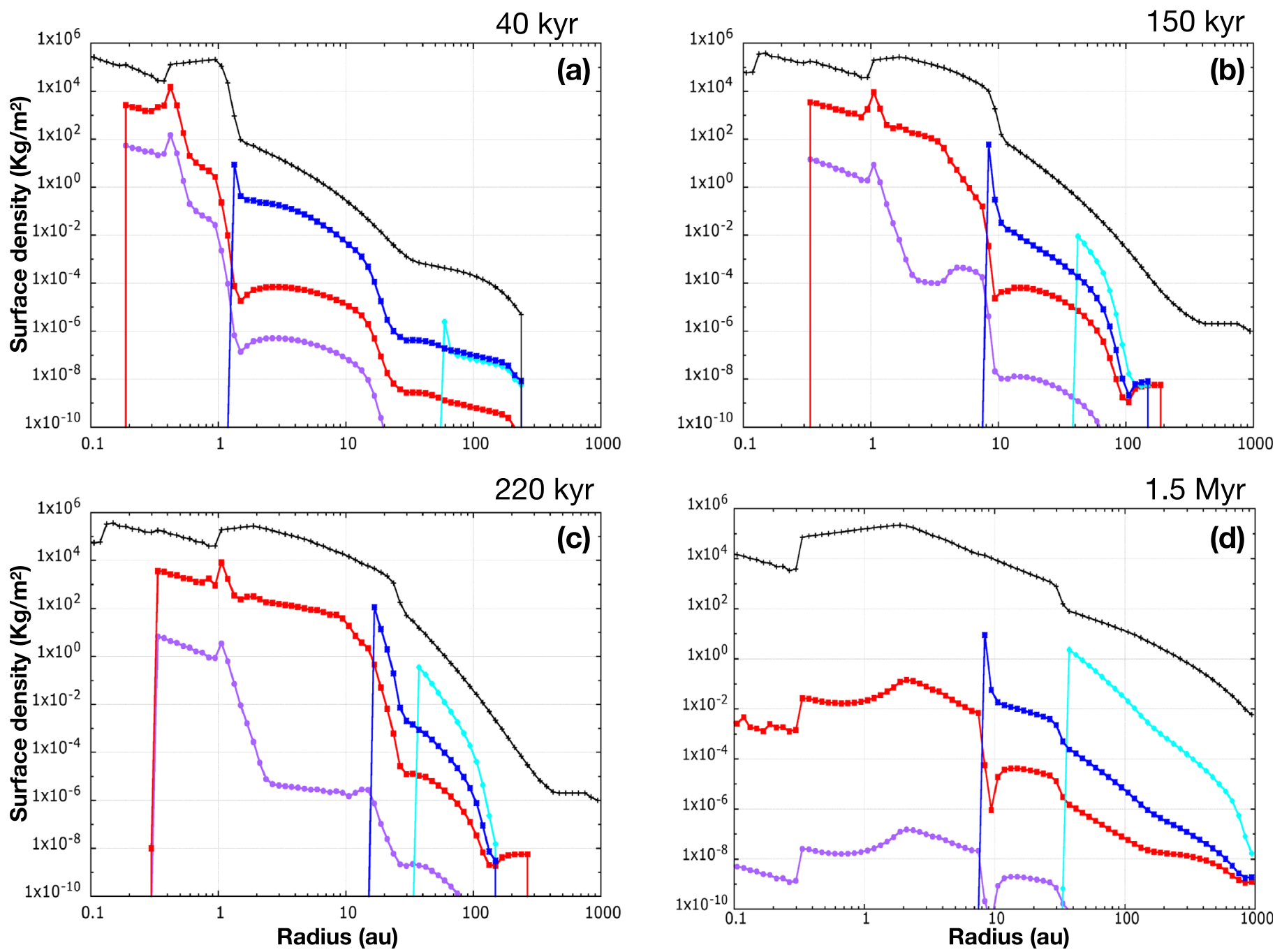

Figure 2. Surface density profiles at four different timescales. $\mathrm{H}_{2}$ (g): black; $\mathrm{H}_{2} \mathrm{O}$ (ice): blue; $\mathrm{CO}$ (ice): cyan; CAIs: purple; other rocky material: red. The disk increases its mass and expands in the early stages ( $\mathrm{a}, \mathrm{b}$, and $\mathrm{c}$, with $\mathrm{c}$ marking the end of the collapse), and then looks like an accretion disk at a later stage (d).

could experience low-temperature chemical alteration $(T<$ $650 \mathrm{~K}$; see Table 1). Later, as $R_{c}(t)$ moves outward, parent cloud dust can be preserved if a given mineral is injected outside of its condensation front. At $t \sim 150 \mathrm{kyr}, R_{c}(t)$ penetrates the DZ (Figure 3(b)) where dust and gas can be stored because of low turbulence. This lowers dramatically the concentration of refractory condensates within the DZ. At the end of the infall, most of the DZ has been fed by parent cloud material, leading to a DZ poor in refractory condensates and a CAIs-enriched outer disk beyond the DZ (Figure 3(c)). Inside of the inner half of the DZ, material can be further thermally processed as the temperature could reach $T \sim 900 \mathrm{~K}$, while the outer half of the DZ contains mostly thermally unaltered dust. Our simulations also show that this heterogeneous dust distribution will be qualitatively preserved over the lifetime of the disk (Figure 3(d)). However, inward from the DZ inner edge $(R<0.3 \mathrm{au})$, the disk becomes CAIs-poor because of strong inward accretion of gas that removes solids.

This distribution predicted for the various dust components in the disk (Figure 3(d)) shows striking similarities with the composition of the three main families of chondritic meteorites (enstatite, ordinary, and carbonaceous chondrites) as defined by their fractions of CAIs, metal, chondrules, and matrix (Scott \& Krot 2003). To perform this comparative exercise, we consider to first order the unprocessed dust as a proxy for the finegrained matrix containing different kinds of circumstellar grains (Nittler 2003) and glass with embedded metal and sulfides (GEMS), a fraction of which are likely surviving interstellar grains (Keller \& Messenger 2011). Within 1-10 au, dust is dominated by disk-born silicates (processed or condensed) and iron (processed or condensed, inside 2 au essentially), and are poor in CAIs and pristine interstellar dust. This is reminiscent of (matrix-poor, chondrule-rich) noncarbonaceous chondrites, i.e., ordinary chondrites and enstatite chondrites (the latter likely formed well inside the water snow line). At larger distances, the dust is dominated by unprocessed interstellar medium (ISM) dust that was injected late when $R_{c}(t)>5 \mathrm{au}$, processed dust, and condensed silicates. This region, though beyond the snow line and thus having water icebearing dust, has the highest fraction of CAIs of the whole disk. This bears similarities to carbonaceous chondrites. Note the presence, in addition to CAIs, of condensed silicates that formed from the same parent gas reservoir. These silicates could then correspond to the Amoeboid Olivine Aggregates of carbonaceous chondrites that are known to have strong links with CAIs (from their oxygen isotopic composition, see Krot et al. 2004). 

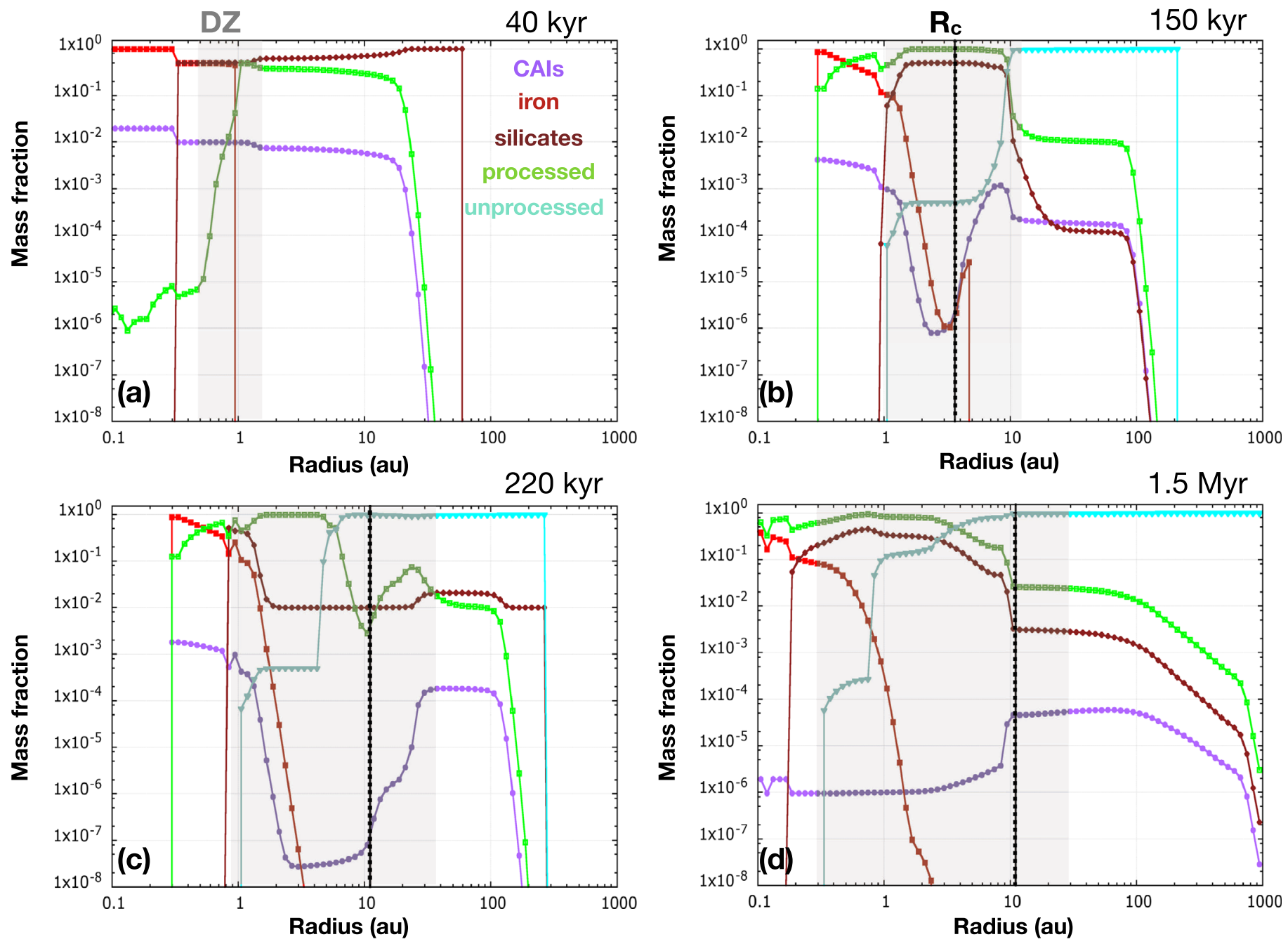

Figure 3. Mass fraction of various types of rocky material in the disk as a function of radius and time. The different types of solids considered are CAIs (purple), metallic iron (red), condensed silicates (brown), all processed dust (green), and all unprocessed dust (cyan). The four panels show the calculated time-integrated dust distribution at four snapshots, using the modeled disk evolution described in Figure 1. The light gray zone shows the extension of the DZ. The black dotted line shows the location of the centrifugal radius $R_{c}(t)$. As $R_{c}(t)$ moves outward, material is injected into the disk at different temperatures, thus experiencing different thermal processing. Similarly to Yang \& Ciesla (2012), the relative decrease of CAIs starting at $t \sim 150 \mathrm{kyr}$ results from a dilution due to the injection of parent cloud material directly from the infalling cloud, which in our case fall into the disk's DZ.

Interestingly, the formation of CAIs is found to be a transient event. In Figure 4(a) we report the time evolution of the CAIs mass in the disk and the time evolution of the accretion rates. In Figure 4(b) we show the time evolution of the temperature in a given location of the disk and the time evolving location of the CAIs' condensation front.

In the simulation, the massive production of CAIs occurs from $t \sim 40 \mathrm{kyr}$ to $t \sim 70 \mathrm{kyr}$, in very good agreement with $\mathrm{Pb}-\mathrm{Pb}$ and ${ }^{26} \mathrm{Al}-{ }^{26} \mathrm{Mg}$ chronologies of CAIs (Connelly et al. 2012; Mishra \& Chaussidon 2014; Figure 4(a)). This corresponds to a short time window, when interstellar solids are injected into the disk at temperatures high enough for them to be fully evaporated (i.e., when $R_{c}(t)$ is inward of the condensation radius of CAIs; see Figure 1), so that upon spreading outward, the gas can condense into CAIs. This time is shorter by a factor of a few than that found by Yang \& Ciesla (2012) because of their lower condensation temperature for refractories.

Still, the production of CAIs appears to occur in steps linked to accretion bursts onto the star following mass loading and instabilities of the DZ (Zhu et al. 2009; Owen \& Armitage 2014;
Figure 4(a)). This may help to explain part of the diversity of CAIs populations across chondrite groups (MacPherson 2014) despite overall derivation from the same reservoir (the inner disk in our model). It is possible that some of the reheating episodes experienced by many CAIs (Richter et al. 2006; Simon et al. 2017) may be linked to such bursts of accretion, reheating, partial vaporization condensations (as suggested in Figure 4), although many of those episodes were likely of much shorter duration. Our results set the formation of the main bulk of CAIs precursors found in chondrites as early as the formation of the disk itself (class 0 to I).

\section{Conclusions}

In this Letter we have investigated the effects of the cloud collapse and disk building in forming and distributing different solids species in the disk. We find that coupling dust transport and thermal processing during the cloud collapse and disk formation naturally results in the local mixing of materials with different thermal histories, in qualitative agreement with the three main chondritic families. Our model also explains the 

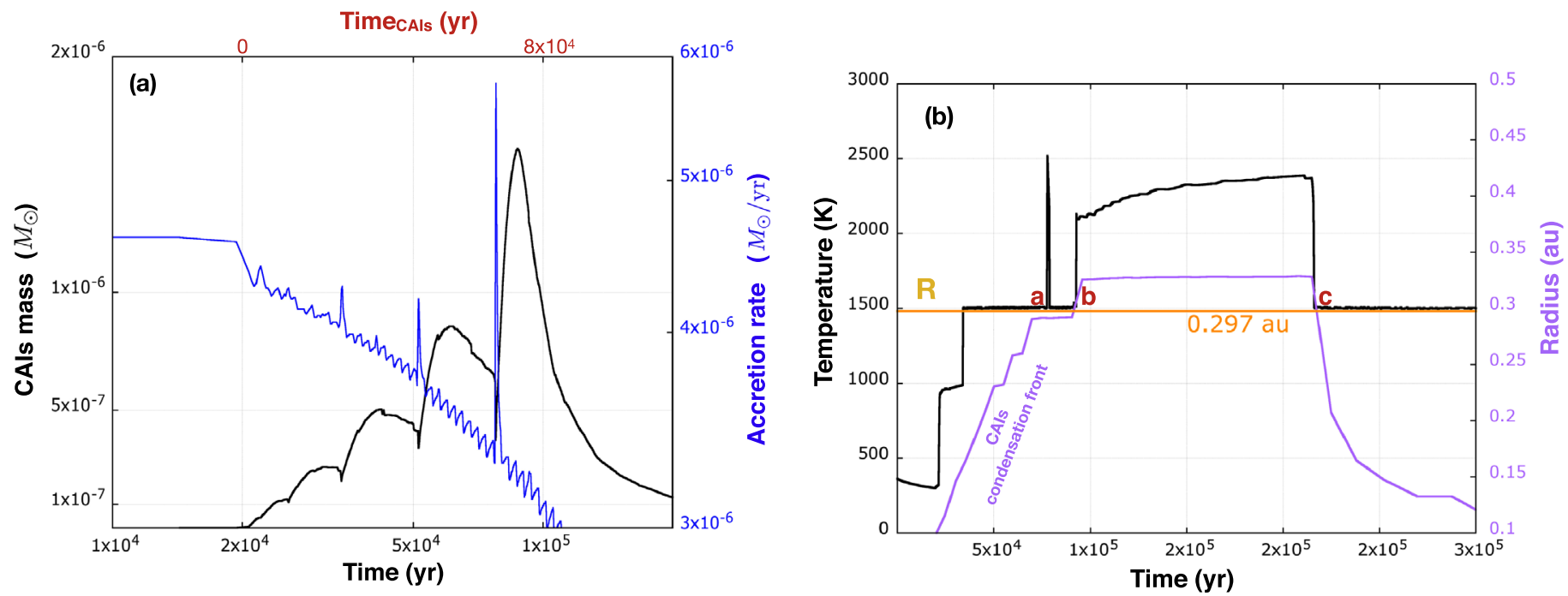

Figure 4. (a): Time evolution of the CAIs mass in the disk (black line, left $y$-axis) and of the accretion rates (blue line, right $y$-axis). The total CAIs mass in the disk is in solar mass and accretion rates in solar mass per year. The zero of the age scale Time ${ }_{\mathrm{CAIs}}$ (upper-horizontal axis) corresponds to the start of CAIs condensation in the simulations. Abrupt steps in the CAIs' mass and accretion rates are observed between 0 and $t \sim 70 \mathrm{kyr}$ (Time ${ }_{\text {CAIs }}$ ). In the simulation, five transient outbursts occur. These events are like "FU Orionis" events (Zhu et al. 2009), and are followed by material evaporation and recondensation. They are caused by mass loading of the DZ due to the gas inflow from the disk and the parent cloud. These episodes are transient because the enhanced viscosity induces a fast spreading of material. Bursts in the production of CAIs occur after episodes of sudden accretion, the strongest one being the one at Time CAIs $_{2} \sim 70$ kyr. (b): Time evolution of the temperature in the disk (black line, left $y$-axis) at a selected radius $(R=0.297 \mathrm{au})$ and the time evolving location of the CAIs' condensation front (purple line, right $y$-axis). The condensation front of CAIs is the location of the $T=1650 \mathrm{~K}$ isotherm (note that after $t \sim 220 \mathrm{kyr}$, when the flow of gas reverses toward the star in this region, this front inverses its displacement as well and moves closer to the star). The temperature profile evidences the presence of an outburst (an abrupt pulse with $T>2000 \mathrm{~K}$ ). If these pulses in temperature take place in a region outward from the condensation front of CAIs, already formed CAIs can experience thermal alteration or partial evaporation (see the point marked "a" in the figure). All of the CAIs that are located at a given radius are vaporized when the CAIs condensation front crosses this radius (marked "b"). CAIs can then recondense as the condensation front moves back toward the inner disk regions (marked " $\mathrm{c}$ "). Note that temperatures as high as those required to evaporate the precursors of Fractionated and Unidentified Nuclear isotopic properties (FUN) CAIs $(T \sim 2200 \mathrm{~K}$ (Mendybaev et al. 2013) can be reached in these outbursts.

overabundance of refractory materials far from the Sun and their short-formation timescales, during the first tens of kyr of the Sun. Our findings can reconcile all of these long-existing cosmochemical questions within one single picture and place the time 0 of the Solar System during the class 0 to I of the Sun's formation history.

Some additional processes (e.g., disk winds acting at the disk surface) may redistribute material at large distances, and a more refined chemistry can give more precise predictions. However, this simple model unveils new ways, never described before, to interpret the distribution of chondritic material in our Solar System, which may have kept the imprint of the could infall. This Letter implies that it is now critical to study the protoplanetary disk, not in isolation, but in the context of the early environment of the Sun, which has left fingerprints in the diversity of our Solar System material.

The authors wish to thank the anonymous referee for the detailed and useful comments that largely improved the manuscript, and Patrick Hennebelle and Yueh-Ning Lee for useful discussions. The authors wish to acknowledge the financial support of ANR-15-CE31-0004-1 (ANR CRADLE) and of the UnivEarthS Labex programme at Sorbonne Paris Citè (ANR-10-LABX-0023 and ANR-11-IDEX-0005-02).

\section{ORCID iDs}

Francesco C. Pignatale 17 https://orcid.org/0000-0003-0902-7421 Sébastien Charnoz (1) https://orcid.org/0000-0002-7442-491X

\section{References}

Asplund, M., Grevesse, N., Sauval, A. J., \& Scott, P. 2009, ARA\&A, 47, 481 Baillié, K., Charnoz, S., \& Pantin, E. 2016, A\&A, 590, A60

Barshay, S. S., \& Lewis, J. S. 1976, ARA\&A, 14, 81

Birnstiel, T., Klahr, H., \& Ercolano, B. 2012, A\&A, 539, A148

Charnoz, S., Pignatale, F. C., Hyodo, R., et al. 2018, A\&A, submitted Chaussidon, M., \& Liu, M.-C. 2015, GMS, 212, 1

Chiang, H.-F., Looney, L. W., \& Tobin, J. J. 2012, ApJ, 756, 168

Connelly, J. N., Bizzarro, M., Krot, A. N., et al. 2012, Sci, 338, 651

Cox, E. G., Harris, R. J., Looney, L. W., et al. 2017, ApJ, 851, 83

Dominik, C., \& Dullemond, C. P. 2008, A\&A, 491, 663

Drążkowska, J., Alibert, Y., \& Moore, B. 2016, A\&A, 594, A105

Dullemond, C. P., Apai, D., \& Walch, S. 2006, ApJL, 640, L67

Ebel, D. S. 2006, in Meteorites and the Early Solar System II, ed. D. S. Lauretta \&

H. Y. McSween Jr. (Tucson, AZ: Univ. Arizona Press), 253

Goldberg, A. Z., Owen, J. E., \& Jacquet, E. 2015, MNRAS, 452, 4054

Helling, C., Winters, J. M., \& Sedlmayr, E. 2000, A\&A, 358, 651

Hennebelle, P., Commerçon, B., Chabrier, G., \& Marchand, P. 2016, ApJL, 830, L8

Henning, T., \& Semenov, D. 2013, ChRv, 113, 9016

Hueso, R., \& Guillot, T. 2005, A\&A, 442, 703

Jacobsen, B., Yin, Q.-z., Moynier, F., et al. 2008, E\&PSL, 272, 353

Jacquet, E., Fromang, S., \& Gounelle, M. 2011, A\&A, 526, L8

Keller, L. P., \& Messenger, S. 2011, GeCoA, 75, 5336

Kimura, S. S., Kunitomo, M., \& Takahashi, S. Z. 2016, MNRAS, 461, 2257

Krot, A. N., Petaev, M. I., Russell, S. S., et al. 2004, ChEG, 64, 185

Larsen, K. K., Trinquier, A., Paton, C., et al. 2011, ApJL, 735, L37

MacPherson, G. J. 2014, in Meteorites and Cosmochemical Processes, ed. A. M. Davis (Amsterdam: Elsevier), 139

Mendybaev, R. A., Richter, F. M., Georg, R. B., et al. 2013, GeCoA, 123, 368

Mishra, R. K., \& Chaussidon, M. 2014, E\&PSL, 390, 318

Nittler, L. R. 2003, E\&PSL, 209, 259

Owen, J. E., \& Armitage, P. J. 2014, MNRAS, 445, 2800 
Pignatale, F. C., Maddison, S. T., Taquet, V., Brooks, G., \& Liffman, K. 2011, MNRAS, 414, 2386

Richter, F. M., Mendybaev, R. A., \& Davis, A. M. 2006, M\&PS, 41, 83

Scott, E. R. D., \& Krot, A. N. 2003, TrGeo, 1, 711

Semenov, D., Henning, T., Helling, C., Ilgner, M., \& Sedlmayr, E. 2003, A\&A, 410,611

Shu, F. H. 1977, ApJ, 214, 488

Simon, J. I., Jordan, M. K., Tappa, M. J., et al. 2017, E\&PSL, 472, 277
Terquem, C. E. J. M. L. J. 2008, ApJ, 689, 532

Turner, N. J., \& Sano, T. 2008, ApJL, 679, L131

Warren, P. H. 2011, GeCoA, 75, 6912

Williams, J. P., \& Cieza, L. A. 2011, ARA\&A, 49, 67

Yang, L., \& Ciesla, F. J. 2012, M\&PS, 47, 99

Zhu, Z., Hartmann, L., \& Gammie, C. 2010, ApJ, 713, 1143

Zhu, Z., Hartmann, L., Gammie, C., \& McKinney, J. C. 2009, ApJ, 701, 620 